\title{
Atividade microbiana no solo em sistema de produção consorciado
}

\author{
Soil microbial activity in a consortium production system \\ Actividad microbiana del suelo en un sistema de producción en consorcio
}

Recebido: 27/10/2021 | Revisado: 03/11/2021 | Aceito: 09/11/2021 | Publicado: 13/11/2021

Hiago Souza Silva
ORCID: https://orcid.org/0000- 0001-7690-9390
E-mail: hiagosouza1128@ @mail.com
Josimar da Silva Chaves
Instituto Federal de Educação, Ciência e Tecnologia de Roraima, Brasil
ORCID: https://orcid.org/0000-0001-5871-5605
Universidade Federal de Roraima, Brasil
E-mail: josimar.chaves@ufrr.br
João Pedro Santos do Nascimento
ORCID:https://orcid.org/0000- 0001-9037-8661
E-mail: jpixeus@gmail.com
Sandoval Menezes de Matos
Instituto Federal de Educação, Ciência e Tecnologia de Roraima, Brasil
ORCID:https://orcid.org/000-0002-3307-1775
E-mail: sandoval.matos@ifrr.edu.br
Alfredo Fernandes de Brito Neto
ORCID: https://orcid.org/0000-0001-8330-9027
Instituto Federal de Educação, Ciência Tecnogia de Roraima, Brasil
E-mail: alfredo_coremas@ hotmail.com
Jadinéa Leandro Leite
Instituto Federal de Educação, Ciência e Tecnologia de Roraima, Brasil
ORCID: https://orcid.org/0000-0003-3210-1474
Instituto Federal de Educação, Ciência e Tecnologia de Roraima, Brasil
E-mail: jadylleite @gmail.com
Hipólito Ribas Pereira
Instituto Federal de Educação, Ciência e Tecnologia de Roraima, Brasil
E-mail: wlligton.agro@gmail.com

\section{Resumo}

As atividades microbianas no solo são importantes indicadores de alterações causadas por práticas agrícolas, sendo responsáveis por transformações físicas, químicas e biológicas nos resíduos orgânicos, que emplicam na fertilidade do solo. Objetivou-se avaliar a atividade microbiana presente no solo em sistema de manejo consorciado com leguminosa, fruteíras e essencia florestal em comparação a uma área de mata nativa. O trabalho foi desenvolvido no Instituto Federal de Ciência e Tecnologia de Roraima - Campus Novo Paraíso, em três áreas, sistema de cultivo consorciado gliricídia + frutíferas (laranja e banana), sem gliricídia (laranja e essenciais florestais) e mata nativa. As amostras de solo foi coletado com profundidade de $0 \mathrm{~cm}-10 \mathrm{~cm}$ e $10 \mathrm{~cm}-20 \mathrm{~cm}$, colocadas em caixa térmica e mantidas a $4{ }^{\circ} \mathrm{C}$. Foi realizado análises do Carbono da Biomassa Microbiana do solo (BMS- C), Nitrogênio da biomassa Microbiana do solo (N-mic) e da população de microrganismos (total, celulíticos, amonificadores e actinobactérias). A área do consórcio de gliricídia + fruteiras (laranja e banana) apresentou maior população de microrganismos e um incremento de $356 \%$ no carbono da biomassa microbiana, mostrando ser um sistema alternativo para o consorcio de espécies vegetais, favorecendo o fornecimento de nutrientes para as plantas.

Palavras-chave: Microbiologia; Diversidade; Agroecologia; Fertilização.

\begin{abstract}
Microbial activities in the soil are important indicators of changes caused by agricultural practices, being responsible for physical, chemical and biological changes in organic residues, which affect soil fertility. The objective was to evaluate the microbial activity present in the soil in a management system intercropped with legumes, fruit trees and forest essence in comparison with an area of native forest. The work was developed at the Federal Institute of Science and Technology of Roraima - Campus Novo Paraíso, in three areas, intercropping system of gliricidia + fruit trees (orange and banana), without gliricidia (orange and forest essentials) and native forest. Soil samples were collected at
\end{abstract}


a depth of $0 \mathrm{~cm}-10 \mathrm{~cm}$ and $10 \mathrm{~cm}-20 \mathrm{~cm}$, placed in a thermal box and kept at $4{ }^{\circ} \mathrm{C}$. Soil Microbial Biomass Carbon (BMS-C), Soil Microbial Biomass Nitrogen (N-mic) and microorganism population (total, cellulite, ammonifiers and actinobacteria) were analyzed. The area of the intercropping of gliricidia + fruit trees (orange and banana) had a greater population of microorganisms and an increase of $356 \%$ in microbial biomass carbon, proving to be an alternative system for the intercropping of plant species, favoring the supply of nutrients for the plants.

Keywords: Microbiology; Diversity; Agroecology; Fertilization.

\section{Resumen}

Las actividades microbianas en el suelo son indicadores importantes de los cambios provocados por las prácticas agrícolas, siendo responsables de los cambios físicos, químicos y biológicos en los residuos orgánicos, que afectan la fertilidad del suelo. El objetivo fue evaluar la actividad microbiana presente en el suelo en un sistema de manejo intercalado con leguminosas, frutales y esencia forestal en comparación con un área de bosque nativo. El trabajo se desarrolló en el Instituto Federal de Ciencia y Tecnología de Roraima - Campus Novo Paraíso, en tres áreas, sistema de cultivos intercalados de gliricidia + árboles frutales (naranjo y plátano), sin gliricidia (naranjos y esenciales forestales) y bosque nativo. Las muestras de suelo se recolectaron a una profundidad de $0 \mathrm{~cm}-10 \mathrm{~cm}$ y $10 \mathrm{~cm}-20 \mathrm{~cm}$, se colocaron en una caja térmica y se mantuvieron a $4{ }^{\circ} \mathrm{C}$. Se analizaron el Carbono de Biomasa Microbiana del Suelo (BMS-C), el Nitrógeno de la Biomasa Microbiana del Suelo (N-mic) y la población de microorganismos (total, celulitis, amonificantes y actinobacterias). El área de intercalación de gliricidia + árboles frutales (naranjo y banano) tuvo una mayor población de microorganismos y un incremento del $356 \%$ en el carbono de biomasa microbiana, demostrando ser un sistema alternativo para la intercalación de especies vegetales, favoreciendo el suministro de nutrientes para las plantas.

Palabras clave: Microbiología; Diversidad; Agroecología; Fertilización.

\section{Introdução}

O uso de sistemas de manejo inadequado pode causar poluição e eutrofização de mananciais, aumentar os custos com adubação e provocar a degradação de agroecossistemas. Os microrganismos presentes no solo são responsáveis por diversos processos que reciclam energia, água e nutrientes do ambiente. Desta forma, o conhecimento da diversidade microbiana e funcional da microbiota adquirem importância no contexto da qualidade do solo, visto que estão associados à estabilidade do ecossistema frente a distúrbios ambientais naturais ou antrópicos (Jurburg \& Salles 2015).

Os atributos microbianos do solo, tais como a diversidade, atividade enzimática, taxa de respiração e biomassa microbiana, são indicadores sensíveis que podem ser utilizados no monitoramento de alterações ambientais decorrentes do uso agrícola, (Epelde et al., 2014; Silva et al., 2021). Os mesmos podem servir para orientar o planejamento e a avaliação das práticas de manejo utilizadas. Algumas das propriedades do solo são resultado da atividade biológicas e as interações entre diversas comunidades de organismos do solo colaboram para o funcionamento do solo, e por diversos outros processos que estão ligados a cadeia tróficas (Santana et al., 2017). A importância dos microrganismos vem sendo discutida pois o mesmo são um dos fatores de formação do solo, e também estão ligados aos processos de decomposição de resíduos orgânicos que resultam na ciclagem dos nutrientes (ciclo biogeoquímicos), na formação da matéria orgânica, e também no sequestro de determinados elementos como o carbono, nitrogênio dentre outros. Dentre os fatores que afetam a comunidade microbiota do solo está a temperatura, manejo do solo, vegetação local, umidade relativa do solo dentre outros (Parkin et al. 1996).

A quantificação da biomassa não fornece indicações sobre os níveis de atividade das populações microbianas do solo, sendo importante também avaliar parâmetros que estimem a atividade microbiana, como a respiração microbiana e atividade enzimática, para verificar o estado metabólico das comunidades de microrganismos do solo (Souto et al., 2009), bem como quantificar grupos de microrganismos que desempenham papel chave na dinâmica da matéria orgânica do solo (Kuzyakov, 2010).

Por estas razões, é justificada a importância do desenvolvimento de pesquisas que envolva o levantamento, por meio de análises microbiológicas da população de microrganismos presente nos solos em diferentes sistemas de manejo, visando a 
sua quantificação, assim como as funções exercidas, objetivando a mitigação dos efeitos das práticas agrícolas adotadas nos sistemas de produção.

\section{Metodologia}

Trata-se de uma pesquisa exploratória de natureza quantitativa (Pereira et al., 2018), realizada no Sul do estado de Roraima, conduzida no Instituto Federal de Educação Ciência e Tecnologia de Roraima - IFRR/Campus Novo Paraíso, localizado nas coordenadas geográficas: latitude $1^{\circ} 15^{\prime} 01,46$ N', longitude $60^{\circ} 29^{\prime} 12,30 \mathrm{~W}$ ', e a uma altitude de 83,09 m, no município de Caracaraí - Roraima. O solo da área é classificado como Latossolo Vermelho distrófico argissólico (Embrapa, 2013).

Os tratamentos foram constituídos de três áreas diferentes: Área 1, sistema de cultivo consorciado (gliricídia (Gliricídia sepium (Jacq.) Steud.) + frutíferas (laranja (Citrus sinensis L. Osbeck), banana (Musa spp.)); Área 2, cultivada com laranja + essência florestal (ipê - Handroanthus heptaphyllus); e Área 3, mata nativa. As áreas foram divididas em cinco parcelas, das quais cada uma foi subdividida em duas subparcelas, em que foram coletadas amostras de solo nas profundidades de $0-10$ cm e $10-20 \mathrm{~cm}$. As amostras foram acondicionadas em sacos plásticos, estéreis, devidamente identificadas, e armazenadas em câmara fria $\left(4^{\circ} \mathrm{C}\right)$ (Sarathchandra, 1978). A microbiota ativa do solo foi avaliada pela técnica de plaqueamento em superfície, em triplicata, utilizando meios específicos de crescimento de microrganismos. As amostras foram homogeneizadas e peneiradas, em seguida retirado $10 \mathrm{~g}$ de solo o qual foi triturado cuidadosamente em um graal esterilizado, depois transferido para um Erlenmeyer contendo $90 \mathrm{~mL}$ de solução salina ( $\mathrm{NaCl}$ 0,85\%), agitada por trinta minutos, em seguida transferido $1 \mathrm{~mL}$ deste solo diluído para tubo de ensaio contendo $9 \mathrm{~mL}$ de solução salina, homogeneizada em agitador do tipo vortex, em diluição seriada decimal conforme citado por Neder, (1992) . Em seguida $0,1 \mathrm{~mL}$ dessa diluição foi transferida para as placas de Petri em triplicata contendo meio de cultura YMA + vermelho congo, citado na literatura como específico para bactérias (Moreira \& Siqueira, 2006), e adicionado antibiótico Cloranfenicol (4 mg/mL), ao meio de cultura para evitar contaminações.

A quantificação foi obtida por unidades formadoras de colônias por grama de solo (UFC g solo ${ }^{-1}$ ). As placas levadas para estufa de crescimento a $28^{\circ} \mathrm{C}$, após 96 horas realizou-se as contagens das unidades formadoras de colônias. Os resultados das repetições empregados para cálculo de médias e erro padrão, foi realizada utilizando o software Microsoft Office Excel 2010 (Microsoft).

A quantificação do número de amonificantes foi utilizado $10 \mathrm{~g}$ de amostra do solo misturadas em solução salina e preparado a partir da diluição $10^{-1}$ sucessivamente até $10^{-5}$. Depois $1 \mathrm{~mL}$ de cada diluição foi transferida para tubo de ensaio contendo meio de cultura específico para amonificadores pela técnica de Sarathchandra (1978). Para a diluição, foram utilizados três tubos de ensaio, contendo $4 \mathrm{~mL}$ de meio de cultura e autoclavados, por 20 minutos. Posteriormente foi adicionado em cada tubo $1 \mathrm{~mL}$ das suspensões de solo; e mantidos, por 5 dias, em sala climatizada $\left(28^{\circ} \mathrm{C}\right)$. A presença de amonificadores foi determinada pela mudança de coloração (da cor laranja para rosa), que foram anotados como positivos (presença) e os sem alteração na coloração como negativo (ausência) de microrganismos amonificadores. A determinação do número mais provável (NMP) foi realizado pela contagem dos tubos que apresentaram a ocorrência de amônia, e o cálculo foi efetuado pela utilização da tabela de probabilidade de ocorrência (Sarathchandra, 1978).

A quantificação de microrganismos celulolíticos foi utilizado $10 \mathrm{~g}$ de amostra de solo diluído em solução salina ( $\mathrm{NaCl}$ $0,85 \%$ ), as diluições em série foram de $10^{-1}$ a $10^{-3}$. Foi transferido $1,0 \mathrm{~mL}$ da suspensão do solo para tubos de ensaio contendo 9,0 mL de meio líquido para organismos celulolíticos, segundo método de Bose (1963). Em cada tubo foi colocado uma tira de papel de filtro esterilizado medindo 7,0 x 1,0 cm, ficando a 2,0 cm acima do nível do meio. As culturas foram incubadas em 
estufa B.O.D à $28{ }^{\circ} \mathrm{C}$ por 37 dias. A estimativa do número de células viáveis foi feita por meio da utilização de tabela do número mais provável método descrito por Sarathchandra (1978).

Para a quantificação de actinobactérias foi usado o método de semeadura em superfície, proposto por Arifuzzamanet et al. (2010). As diluições em série foram de $10^{-2}$ a $10^{-6}$, transferido $0,1 \mathrm{~mL}$ da suspensão do solo para as placas de Petri contendo meio de cultura caseína-dextrose-ágar (Clark, 1965). As culturas foram incubadas em estufa B.O.D à $28^{\circ} \mathrm{C}$ por 7 dias. Após este período foram selecionadas e quantificadas as diluições que apresentaram entre 30 a 300 colônias e o resultado expresso em UFC g solo-1 (Dionísio et al., 2016).

Para a determinação do Carbono da Biomassa Microbiana do solo (BMS-C), foi utilizado o método fumigaçãoextração proposto por Vance et al. (1987) e Tate et al. (1988). Para a determinação da respiração basal (RBS), que consiste na análise de $\mathrm{CO}_{2}$ produzido pelos microrganismos, através da degradação da matéria orgânica (MOS) as amostras recém coletadas e peneiradas, foram analisadas em duplicata, para isto a amostra inicial foi dividida em três sub-amostras de $50 \mathrm{~g}$ acondicionadas em frascos de vidro de $100 \mathrm{~mL}$, sendo uma delas para determinação da umidade do solo e as demais para a respiração basal do solo, utilizando a metodologia proposta por Jenkinson \& Powlson (1976).

Para a determinação de Nitrogênio da biomassa Microbiana do solo (BMS-N), tendo em vista que o Nitrogênio (N) é o segundo elemento mais abundante do corpo microbiano, é de suma importância determina-lo, o método utilizado foi o de fumigação-extração, com a relação de solo extrator de 1:2,5 (Tate et al 1988), com a adição de clorofórmio diretamente na amostra, mantendo as em local escuro por 24 horas, dando prosseguimento a extração e a quantificação do nitrogênio microbiano por destilação de arraste de vapor (Kjeldanhl), seguida de volumetria de neutralização ácido-base. As análises químicas e biológicas do solo, foram realizadas no Laboratório de Solos e Planta do IFRR - Campus Novo Paraíso.

Os dados das variáveis obtidas em cada época de amostragem do solo, distintamente, foram submetidos a análise de variância e os resultados das médias de cada tratamento foram analisados estatisticamente utilizando o programa SISVAR, versão 4.3 (Ferreira, 2014). Os efeitos dos tratamentos foram comparados pelo teste de Tukey a 5\% de probabilidade.

\section{Resultados e Discussão}

Nos dados obtidos (Tabela 1) após 96h de incubação, a área com presença de glirícidia + fruteiras apresentou um

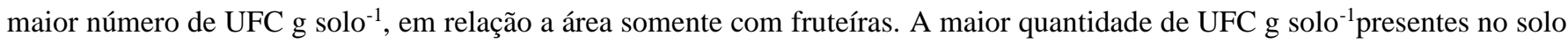
com plantas em consórcio com gliricídia, é explicada por essa leguminosa apresentar altos teores de nutrientes, principalmente nitrogênio, fixado por microrganismos simbióticos, constituindo uma alternativa viável para o fornecimento de nutrientes para as espécies frutíferas (Espindola et al., 2006; Leal et al., 2021; Matos et al., 2021). Observa-se que o número de unidades formadoras de colônias (UFC g solo ${ }^{-1}$ ), nos três ambientes estudados, foram sofrendo redução conforme o aumento da profundidade. $\mathrm{O}$ solo apresenta pH baixo (ácido), o qual afeta a população microbiana e a solubilidade dos minerais no solo, afetando, portanto, a disponibilidade de nutrientes. 
Tabela 1 - População de microrganismos (total, celulíticos, amonificadores e actinobactérias) nas áreas do estudo

\begin{tabular}{|c|c|c|c|c|c|c|}
\hline Área coletada & $\begin{array}{l}\text { Profundidade } \\
\qquad(\mathrm{cm})\end{array}$ & $\mathrm{pH}$ & $\begin{array}{c}\text { Microrganismos } \\
\text { total } \\
\text {................................ }\end{array}$ & $\begin{array}{l}\text { Celuliticos } \\
. . U F C g \text { solo } \\
\end{array}$ & Amonificadores & Actinobactérias \\
\hline \multirow{2}{*}{$\begin{array}{c}\text { Gliricídia + laranja }+ \\
\text { banana }\end{array}$} & $0-10$ & 6.13 & $3,0 \times 10^{4}$ & $11,0 \times 10^{3}$ & $14,0 \times 10^{4}$ & $3,6 \times 10^{3}$ \\
\hline & $10-20$ & 6.07 & $3,5 \times 10^{4}$ & $2,5 \times 10^{3}$ & $14,0 \times 10^{4}$ & $3,1 \times 10^{3}$ \\
\hline \multirow{2}{*}{ Laranja + Ipê } & \multirow{2}{*}{$0-10$} & 6.00 & $2,4 \times 10^{6}$ & $0,4 \times 10^{3}$ & $14,0 \times 10^{4}$ & $3,0 \times 10^{3}$ \\
\hline & & 6.09 & $1,7 \times 10^{6}$ & $0,35 \times 10^{3}$ & $11,0 \times 10^{4}$ & $*$ \\
\hline \multirow{2}{*}{ Mata Nativa } & $0-10$ & 4.57 & $6,8 \times 10^{5}$ & $11,0 \times 10^{3}$ & $*$ & $*$ \\
\hline & $10-20$ & 4.76 & $2,2 \times 10^{6}$ & $11,0 \times 10^{3}$ & $0,07 \times 10^{4}$ & $*$ \\
\hline
\end{tabular}

*não houve crescimento de microrganismos.

Análise realizada no Laboratório de Solos e Planta do IFRR, Caracaraí-RR, 2021.

Fonte: Autores.

O solo da área de mata apresentou uma maior quantidade de UFC g solo ${ }^{-1}$ na profundidade de $0-10 \mathrm{~cm}$. A atividade biológica é altamente concentrada nas primeiras camadas do solo, na profundidade entre 1 a $30 \mathrm{~cm}$. Nestas camadas, o componente biológico ocupa uma fração de menos que $0,5 \%$ do volume total do solo e representa menos que $10 \%$ da matéria orgânica. Este componente biológico consiste principalmente de microrganismos que realizam diversas funções essenciais para o funcionamento do solo.

Em relação a presença de microrganismos amonificadores, duas áreas avaliadas apresentaram resultados semelhantes (consórcio gliricídia + laranja + banana e laranja + Ipê) (Tabela 1). Sendo que, a área da mata nativa apresentou menor valor. Segundo Ferreira et al. (2017), os sistemas de cultivo provocam alterações nos atributos microbiológicos do solo quando comparados com a mata, usada como referência. O maior valor de UFC g solo ${ }^{-1}$ presente no solo com plantas em consorcio com gliricídia é explicada por tal leguminosa favorecer a fixação de $\mathrm{N}_{2}$ para as espécies frutíferas, tendo em vistasua capacidade de fixar biologicamente o nutriente, (Kaba et al, 2019).

A população celulolítica foi observada nas três áreas avaliadas (Tabela 1). A área de mata nativa mostrou valores maiores sem diferença nas profundidades. Mesmo existindo uma tendencia de ecossistemas estáveis como o solo sob a mata nativa possuir uma maior diversidade da comunidade microbiana, o solo do consórcio gliricídia + laranja + banana apresentou valor igual a mata nativa na profundidade de $0-10 \mathrm{~cm}$. Para Ramos et al. (2012) este grupo de microrganismos é fortemente influenciado pela cobertura vegetal do local, deste modo, área do consórcio gliricídia + laranja + banana e a mata nativa desempenham papéis semelhantes quanto a disponibilidade no solo do substrato celulose, ambas possuem uma cobertura vegetal maior que a da área de laranja e ipê.

O número de organismos actinobactérias foi maior na amostra de solo na área consórcio gliricídia + laranja + banana, com valores semelhantes nas duas profundidades. Enquanto, na área com laranja + ipê, apenas na profundidade de 10-20cm, o resultado não diferiu com a área consórcio gliricídia + laranja + banana. Enquanto a área da mata não apresentou nenhum crescimento (Tabela 1).

Em relação ao Carbono da Biomassa Microbiana do Solo (BMS-C), houve um incremento de 356\% na área gliricídia + laranja + banana, em relação a área com laranja + Ipê, na profundidade de 0-10 cm (Tabela 2). Esse resultado demostra como citado por Silva et al. (2021), maior atividade microbiológica na camada superficial do solo em virtude do maior teor de matéria orgânica presente nesta. Outro fator que justifica esse incremento é o manejo aplicado nesta área com as podas das plantas de gliricídia sendo a biomassa colocadas na região da rizosfera das plantas de laranja. 
Tabela 2 - Valores de Carbono da Biomassa Microbiana do Solo (BMS-C) e Nitrogênio microbiano (N-mic) coletados em três ambientes diferentes no período seco do ano.

\begin{tabular}{ccllc}
\hline Área & $\begin{array}{c}\text { Profundidade } \\
(\mathrm{cm})\end{array}$ & $\mathrm{pH}$ & \multicolumn{2}{c}{$\begin{array}{c}\mathrm{BMS}-\mathrm{C} \\
\ldots \ldots \ldots . \mathrm{mg} \mathrm{kg} \mathrm{solo} \mathrm{m}^{-1} \ldots \ldots . . .\end{array}$} \\
\hline Gliricídia + laranja + banana & $0,0-10$ & 6.13 & $989,60 \mathrm{a}$ & $347,34 \mathrm{a}$ \\
& $10-20$ & 6.07 & $507,15 \mathrm{a}$ & $99,72 \mathrm{~b}$ \\
\hline Laranja + Ipê & $0,0-10$ & 6.00 & $277,77 \mathrm{~b}$ & $314,10 \mathrm{a}$ \\
& $10-20$ & 6.09 & $404,29 \mathrm{~b}$ & $159,54 \mathrm{a}$ \\
\hline Mata nativa & $0,0-10$ & 4.57 & $1074.7 \mathrm{a}$ & $236,4 \mathrm{~b}$ \\
& $10-20$ & 4.76 & $442,7 \mathrm{~b}$ & $35,3 \mathrm{c}$ \\
\hline
\end{tabular}

Médias seguidas de mesmas letras na coluna, não diferem entre si teste de Tukey a 5\% de probabilidade.

Análise realizada no Laboratório de Solos e Planta do IFRR, Caracaraí-RR, 2021.

Fonte: Autores.

Em relação ao nitrogênio microbiano $\left(\mathrm{N}_{\text {mic }}\right)$, não foi observado diferença significativa entre os sistemas de manejo do solo nas nas áreas com gliricídia + laranja + banana e com laranja + Ipê, na profundidade de 0-10 cm. Porém, essas duas áreas demonstraram valores superiores em relação a área de mata nativa.

As interações bióticas e abióticas que ocorrem em um sistema de manejo, podem interferir nos parâmetros microbiológicos do solo. Tomando como base os resultados observados para os atributos do Carbono da biomassa microbiana (BMS-C), pode ser inferir que, as áreas de consórcio gliricídia + laranja + banana e mata nativa, em relação a área do consórcio laranja +ipê, promovem mais incorporação de matéria orgânica ao solo, proporcionando maior valor de Carbono da biomassa microbiana (BMS-C) na camada de $0-10 \mathrm{~cm}$.

\section{Conclusão}

A área do consórcio de gliricídia com plantas frutiferas (laranja e banana), apresentou um maior valor de unidades formadoras de colônias, proporcionando um maior valor de população de microrganismos (total, celulíticos, amonificadores e actinobactérias), incremento na biomassa microbiana do solo, além de ajudar no fornecimento de nitrogênio para as espécies. Os sistemas de cultivo provocam alterações nos atributos microbiológicos do solo quando comparados com a condição preservada do ambiente natural (mata nativa).

\section{Agradecimentos}

Ao Instituto Federal de Educação, Ciência e Tecnologia de Roraima - IFRR/Campus Novo Paraíso e ao Núcleo de Estudo, Pesquisa, Extensão em Agroecologia (NEPEAGRO) pela disponibilização da área do experimento.

\section{Referências}

Arifuzzamanet, M. R. \& Khatun and H. (2010). Rahman. Isolation and screening of actinomycetes from Sundarbans soil for antibacterial activity. African Journal of Biotechnology Vol. 9(29), pp. 4615-4619, 19 July, AJB ISSN 1684-5315. https://doi.org/10.5897/AJB10.339

Bose, R. A. (1963). Modified Cellulosic Medium for the Isolation of Cellulolytic Fungi from Infected Materials and Soils. Nature 198, 505-506 https://doi.org/10.1038/198505a0

Cherubin, M.R.; Eitelwein, M.T.; Fabbris, C.; Weirich, S.W.; Silva, R.F.; Silva,V.R.; \& Basso, C.J. (2015). Qualidade física, química e biológica de um Latossolo com diferentes manejos e fertilizantes. Revista Brasileira Ciência do Solo, 39: 615-625.

Clark, F. E. Agarplate method for total microbial count. In: Black, C. A., Evans, D. D., Ensminger, L. E., White, J. L. \& Clark, F. E. (1965). Methods of soil analysis. Madison: American Society of Agronomy. https://doi.org/10.2134/agronmonogr9.2.c48

Dionisio J. A. et al. (2016). Guia prático de biologia do solo. Curitiba:SBCS:NEPAR, 152 p. il. https://ainfo.cnptia.embrapa.br/digital/bitstream/item/142645/1/Diana-Signor-guia-pratico-biologia-solo.pdf. ISBN 978-85-69146-00-1. 
EMBRAPA (2013). Sistema Brasileiro de Classificação de Solos / Humberto Gonçalves dos Santos ... [et al.]. - 3 ed. rev. ampl. - Brasília, DF : Embrapa, 2013. $353 \mathrm{p}$.

Epelde, L. et al. (2014). Microbial properties and attributes of ecological relevance for soil quality monitoring during a chemical stabilization field study. Applied Soil Ecology, v. 75, p. 1-12.

Espindola, J. A. A.; Guerra, J.G.M.; Perin, A.; Teixeira, M.G.; Almeida, D.L.; Urquiaga, S.; \& Busquet, R.N.B. (2006). Bananeiras consorciadas com leguminosas herbáceas perenes utilizadas como coberturas vivas. Pesquisa agropecuária brasileira. Brasília. 41 (3), 415 - 420.

Ferreira, D. F. (2014). Sisvar: a computer statistical analysis SYSTEM. Ciência Agrotecnologia - UFLA. 35(6). 1039 - 1042.

Jurburg, S.D \& Salles, J.F. (2015). Functional redundancy and ecosystem function - The soil microbiota as a case study. In: LO YH et al. Biodiversity in ecosystems - linking structure and function. Rijeka: Intech. p. 29-49.

Kaba, J.S., Zerbe, S., Agnolucci, M. et al. (2019). Atmospheric nitrogen fixation by gliricidia trees (Gliricidia sepium (Jacq.) Kunth ex Walp.) intercropped with cocoa (Theobroma cacao L.). Plant Soil 435, 323-336. https://doi.org/10.1007/s11104-018-3897-x

Leal, M. L. A.; Chaves, J.S.; Silva, J.A.; Silva, L.S.; Soares, R.B.; Nascimento, J.P.S.; Matos, S. M.; Teixeira Júnior, D.L.; \& Brito Neto, A.F. (2021). Efeito dos sistemas de manejo e do uso do solo na população de microrganismos do solo. Research, Society and Development, v. 10 , n. 9, e21910917966. http://dx.doi.org/10.33448/rsd-v10i9.17966

Matos, S. M., Alves, R.N.; Chaves, J.S.; Soares, R.B.; Nascimento, J.P.S.; Silva, L.S.; Leal, M. L. A.; Marzano, I.M.; Moraes, G.S.C.; \& Souza, F.G. (2021). Efeitos do uso de gliricídia e rocha fosfatada no crescimento e nos teores de N, P e K nas culturas do quiabo e pepino. Research, Society and Development, v. 10, n. 4, eXX, http://dx.doi.org/10.33448/rsd-v10i4.XXXXX

Moreira, F. M.S. \& Siqueira, J. O. (2006). Microbiologia e Bioquímica do Solo. 2.ed. atual.e ampl.—Lavras:Editora UFLA, 729 p.

Kuzyakov, Y. (2010). Priming effects: Interactions between living and dead organic matter. Soil Biology and Biochemistry, v. 42, n. 9, p. 1363-1371.

Oliveira, L.G.; Batalha, M.O.; \& Pettan, K.B. (2017). Comparative assessment of the food purchase program and the national school feeding program's impact in Ubá, Minas Gerais, Brazil. Ciência Rural. Santa Maria, 47: 01- 06.

Parkin, T.B.; Doran, J.W. \& Francop-Vizcaino, E. (1996). Field and laboratory tests of soil respiration. In: Doran, J.W. \& Jones, A. (Eds) - Method for assessing soil quality. Madison, Soil Science Society of America, p. 231-245.

Pereira A. S. et al. (2018). Metodologia da pesquisa científica. [free e-book]. Santa Maria/RS. Ed. UAB/NTE/UFSM

Santana, A C; Chaves, J.S; \& Rodriguez, C.A. (2017). Biomassa microbiana em diferentes sistemas de manejo do solo no sul do estado de Roraima. Revista Brasileira de Ciências da Amazônia, p.1-67.

Sarathchandra, S.U. (1978). Nitrification activities and the changes in the populations of nitrifying bacteria in soil perfused at two different H-ion concentrations. Plant Soil 50, 99-111. https://doi.org/10.1007/BF02107160

Silva, C. F. et al. (2021). Soil attributes as indicators of the stabilization process of erosion in gullies at different formation stages in the southeast region of Brazil. Revista Ambiente \& Água [online]. 2021, v. 16, n. 4. Epub 04 Aug ISSN 1980-993X. https://doi.org/10.4136/ambi-agua.2632.

Souto, P.C.; Bakke, I.A.; Souto, J.S. \& Oliveira, V.M. (2009) - Cinética da respiração edáfica em dois ambientes distintos no semiárido da Paraíba, Brasil. Revista Caatinga, vol. 22, n. 3, p. 52-58.

Tate, K.R.; Ross, D.J.; \& Feltham, C.W. (1988). A direct extraction method to estimate soil microbial C: Effects of experimental variables and some different calibration procedures. Soil Biol. Biochem., 20:329-335.

Vance, E.D.; Brookes, P.C.; \& Jenkinson, D.S. (1987). An extraction method for measuring soil microbial biomass C. Soil Biology and Biochemistry. 19: 703707. 